\title{
Synthesis of Cross-Coupled Filters With Frequency-Dependent Couplings
}

\author{
Stefano Tamiazzo and Giuseppe Macchiarella, Fellow, IEEE
}

\begin{abstract}
This paper presents a novel synthesis technique for coupled-resonator filters with frequency-dependent couplings. Unlike the works so far appeared in the literature, the proposed technique is not based on the optimization of a network with assigned topology, but it consists in the explicit synthesis of a folded prototype with frequency-dependent transversal couplings. The proposed procedure starts with the synthesis of a new type of canonical prototype with frequency-invariant couplings (the asymmetric lattice); this prototype is then suitably transformed by means of scaling and rotations of coupling and capacitance matrices for obtaining the new configuration with frequency-dependent couplings. Concerning the asymmetric lattice prototype, we show how this canonical network degenerates, in a special case, into the cul-de-sac form; this happens when the assigned reflection zeros are imaginary (or in pairs with opposite real part). This also implies that cul-de-sac is admissible only when reflection zeros satisfy this condition. The novel synthesis approach is illustrated with several examples. A test diplexer employing filters with frequency-dependent couplings has been designed and fabricated for validating the novel synthesis approach.
\end{abstract}

Index Terms-Coupling matrix synthesis, frequencydependent couplings, generalized Chebyshev filters.

\section{INTRODUCTION}

$\mathbf{T}$ HE design of microwave filters exhibiting transmission zeros is today faced with well established procedures [1]-[3] that typically start with the synthesis of a low-pass prototype network composed of ideal lumped components (inverter and capacitors/inductors). The most used configurations are those based on cross-coupled topologies, because they allow flexibility and compactness in the final implementation. However, new solutions are being considered in the last years in order to further reduce the overall size and facilitate the implementation of high-selectivity microwave filters in an integrated environment. In this direction are the works recently appeared in the literature concerning the implementation of transmission zeros by means of frequencydependent couplings [4]-[14]. Some works [4], [5] actually introduce the frequency variation of the couplings in order to develop a more accurate modeling of real filters (allowing a more accurate design, especially in the case of moderate

Manuscript received May 31, 2016; revised October 13, 2016; accepted November 19, 2016. Date of publication December 13, 2016; date of current version March 3, 2017.

S. Tamiazzo is with CommScope, 20864 Agrate Brianza, Italy (e-mail: stefano.tamiazzo@commscope.com).

G. Macchiarella is with the Dipartimento di Elettronica Informazione e Bioingegneria, Politecnico di Milano, 20133 Milan, Italy (e-mail: giuseppe.macchiarella@polimi.it).

Color versions of one or more of the figures in this paper are available online. and large bandwidth). More recently, the use of frequencyvariable couplings has been exploited for introducing transmission zeros in the frequency characteristic. Amari and Bornemann [6] propose the use of resonant inverters in inline filters for getting imaginary transmission zeros. A similar solution is also presented in [7]; in both these works, the filters are initially synthesized assuming ideal inverters and optimization is then used for taking into account the resonating characteristic of the couplings. Another group of works investigates the possibility of realizing cross-coupled filters with some frequency-dependent couplings [8]-[14]. In this case, the implementation of the zeros is not directly related to the resonance of a coupling but just on its variation with frequency (always assumed linear). Combining cross-couplings with frequency-dependent couplings allows reducing the overall number of couplings for a given set of transmission zeros. Moreover, it is shown in [4] that any prototype network with couplings linearly variable in frequency can be transformed into a new network with constant couplings. This means that, starting from the characteristic polynomials defining the generalized Chebyshev characteristic [1], it must be possible to synthesize a network with variable couplings exhibiting such characteristic. The point, however, is that, unlike the prototype with constant couplings, there is no general synthesis technique allowing the design of a prototype including frequency-dependent couplings. For this reason, all the works in the literature on this topic [4]-[14] approach the design of a prototype with assigned topology (and number of frequencyvariant couplings) by means of optimization. In addition, also the choice of the topology represents an open point: in fact, the minimum path rule [15] does not hold for networks with frequency-dependent couplings. Consequently, we are not able to know, before the optimization completes successfully, if a network with assigned topology and frequency-variant couplings can be actually synthesized.

In order to overcome the mentioned drawbacks, we present, in this paper, a novel synthesis procedure for general low-pass prototype with folded topology and transversal cross-couplings linearly variable with frequency [16]. In Sections II and III, we introduce and discuss the asymmetric lattice network, a prototype with frequency-independent couplings, which is a generalization of the symmetric lattice proposed by Bell [17]. In particular, the strict relationship between this topology and cul-de-sac form introduced by Cameron et al. [18] is pointed out. Then, a procedure for transforming the asymmetric lattice network into a canonical prototype with frequency-variable couplings is described. In Section IV, several synthesis examples, which illustrate the 


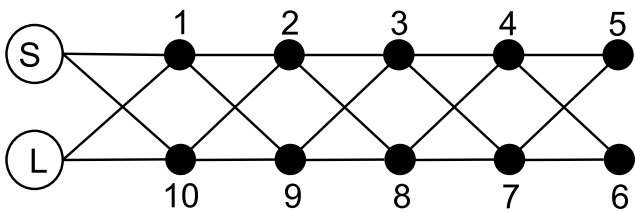

(a)

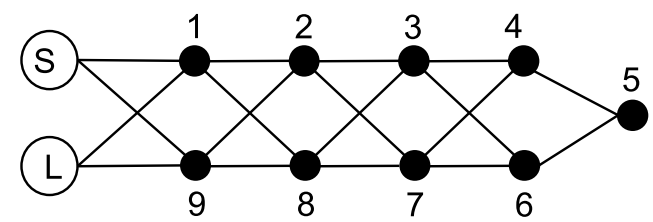

(b)

Fig. 1. Asymmetric lattice network. (a) Even order. (b) Odd order. The solid lines represent ideal admittance inverters and the black nodes are unit capacitances in parallel to frequency-invariant susceptances. Coupling $S-L$ (not reported here) is requested when the number of transmission zeros is equal to the number of poles.

features of the proposed procedure and its advantages, are presented. Finally, Section V describes how to approach the implementation of frequency-variable couplings and reports an example of a fabricated diplexer including frequency-variable couplings, designed with the proposed technique.

\section{Synthesis OF ThE AS yMmetric LAtTiCE Network}

The asymmetric lattice network here introduced is shown in Fig. 1. Unlike the symmetric one proposed by Bell [17], the asymmetric lattice has unequal oblique cross couplings in each lattice block, while the vertical couplings are absent. Note that we have not included the coupling $S$ - $L$, which is, however, required for a fully canonical characteristic.

To synthesize the asymmetric lattice, we start with the evaluation of the characteristic polynomials defining the two-port $S$ matrix of the filter (many methods are available in the literature [1])

$$
S=\left[\begin{array}{cc}
F(s) & P(s) \\
P(s) & (-1)^{N p} F^{*}(s)
\end{array}\right] / E(s) .
$$

Using the polynomials $F, P$, and $E$, we synthesize the folded canonical prototype [1], represented by the coupling matrix $M$. Then, the lattice topology is obtained with a sequence of rotations of $M$ that annihilate all the vertical cross couplings. Assuming $[i, j]$ as the cross-pivots coordinates, the rotation angles are given by

$$
\begin{aligned}
\theta_{k} & =\frac{1}{2} \tan ^{-1}\left(\frac{2 M_{i j}}{M_{j j}-M_{i i}}\right) \quad k=1 \ldots m, i, j=0 \ldots N p+1 \\
m & =\frac{N p}{2}, \quad i=m+1-k, \quad j=m+k(N p \text { even }) \\
m & \left.=\frac{N p-1}{2}, \quad i=m+1-k, j=m+k+1 \text { ( } N p \text { odd }\right) .
\end{aligned}
$$

Note that these rotations are the same used in [1, p. 338] for generating the cul-de-sac configuration in the case of $N p$ even. This means that there is a strict relation between the asymmetric lattice and the cul-de-sac. In fact, if the roots of $F(s)$ are imaginary or in para-conjugate pairs, the asymmetric lattice degenerates into a cul-de-sac form.

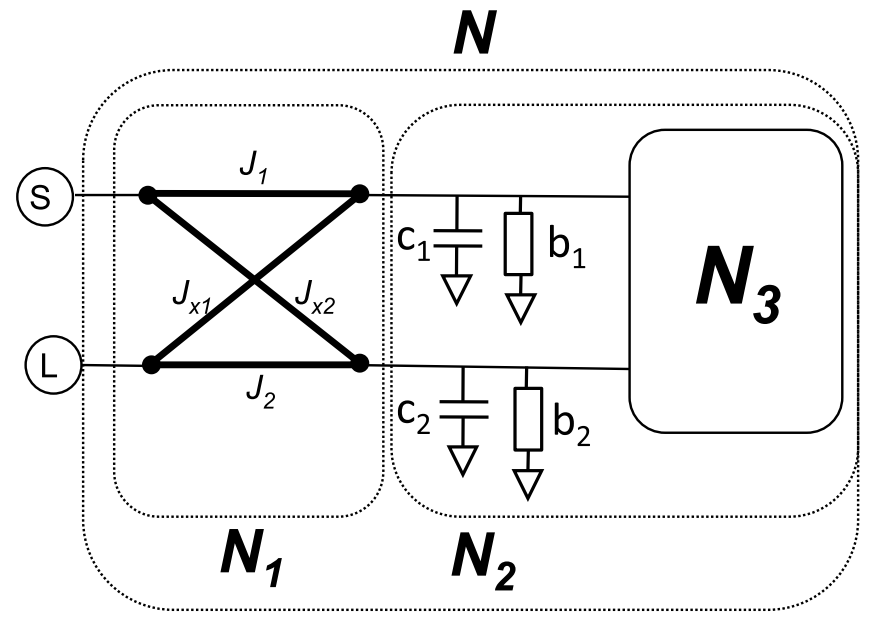

Fig. 2. Synthesis by extraction of the lattice network. Each thick line in network $N_{1}$ is an ideal admittance inverter with parameter $J_{k}$.

\section{A. Synthesis of the Asymmetric Lattice by Extractions}

For demonstrating the last statement, an alternative approach to the synthesis of the asymmetric lattice is used. The synthesis is carried out through the iterative extraction of a basic lattice block constituted by four cross-coupled admittance inverters and two admittances $Y_{1}$, and $Y_{2}$ (Fig. 2). Note that this extraction involves input and output ports simultaneously.

The extractions are performed on the $Y$ matrix of the filter, which is defined as

$$
Y=\left[\begin{array}{cc}
n_{1}(s) & -P(s) \\
-P(s) & n_{2}(s)
\end{array}\right] / d(s)
$$

with

$$
\begin{aligned}
n_{1} & =\frac{1}{2}\left[E-(-1)^{N p} E^{*}+(-1)^{N p} F^{*}-F\right] \\
n_{1} & =\frac{1}{2}\left[E-(-1)^{N p} E^{*}-(-1)^{N p} F^{*}+F\right] \\
d & =\frac{1}{2}\left[E+(-1)^{N p} E^{*}+(-1)^{N p} F^{*}+F\right] .
\end{aligned}
$$

Without loss of generality, in the following, it is assumed that the source-load direct coupling is already removed, and therefore the degree of polynomial $P$ is at most $N p-1$. It is also assumed that no frequency-invariant susceptance to ground is present at source and load nodes. Under these conditions, the degree of polynomial $d$ is $N p$, that of polynomials $n_{1}$ and $n_{2}$ is $N p-1$. The four-port network $N_{\mathbf{1}}$ in Fig. 2 is comprised of four admittance inverters connecting the source and load ports to the input and output ports of the remainder network $\boldsymbol{N}_{\mathbf{2}}$. Its admittance matrix $Y_{J}$ is thus given by

$$
Y_{J}=j\left[\begin{array}{cccc}
0 & 0 & J_{1} & J_{x 1} \\
0 & 0 & J_{x 2} & J_{2} \\
J_{1} & J_{x 2} & 0 & 0 \\
J_{x 1} & J_{2} & 0 & 0
\end{array}\right] .
$$

Without loss of generality, we impose $J_{1}=J_{2}=1$, which sets the level of admittance of the network. After some algebraic manipulations, it is found that the elements of the $Y$ matrix 
of remainder network $N_{2}$ can be expressed as

$$
\begin{aligned}
n_{1}^{\prime} & =n_{2}+J_{x 2}^{2} n_{1}+2 J_{x 2} p \\
n_{2}^{\prime} & =n_{1}+J_{x 1}^{2} n_{2}+2 J_{x 1} p \\
-P^{\prime} & =\left(1+J_{x 1} J_{x 2}\right) p+J_{x 1} n_{2}+J_{x 2} n_{1} .
\end{aligned}
$$

The goal of the extraction is to remove two transmission zeros from network $N$. We then choose $J_{x 1}$ and $J_{x 2}$ so that the degree of $P^{\prime}$ is dropped down to $N p$-3, i.e., the coefficients of degree $N p$-1 and $N p$-2 of polynomial $P^{\prime}$ (here denoted as $p_{(N-1)}^{\prime}$ and $\left.p_{(N-2)}^{\prime}\right)$ vanish

$\left\{\begin{array}{l}\left(1+J_{x 1} J_{x 2}\right) p_{(N-1)}+J_{x 1} n_{2,(N-1)}+J_{x 2} n_{1,(N-1)}=0 \\ \left(1+J_{x 1} J_{x 2}\right) p_{(N-2)}+J_{x 1} n_{2,(N-2)}+J_{x 2} n_{1,(N-2)}=0 .\end{array}\right.$

The next step is the extraction of $\left(c_{1}, b_{1}\right)$ and $\left(c_{2}, b_{2}\right)$ at the input and output of the network $\mathrm{N}_{2}$.

Let $Y_{1}(s)=c_{1} s+j b_{1}$ and $Y_{2}(s)=c_{2} s+j b_{2}$ be the admittances to be removed. The elements of the $Y$ matrix of remainder network $\boldsymbol{N}_{\mathbf{3}}$ are then given by

$$
\begin{aligned}
n_{1}^{\prime \prime} & =n_{1}^{\prime}-Y_{1}(s) \cdot d^{\prime} \\
n_{2}^{\prime \prime} & =n_{2}^{\prime}-Y_{2}(s) \cdot d^{\prime} \\
P^{\prime \prime} & =P^{\prime} \\
d^{\prime \prime} & =d^{\prime} .
\end{aligned}
$$

For reducing the degree of network $N_{\mathbf{3}}$ by 2, we choose the four parameters $c_{1}, b_{1}, c_{2}$, and $b_{2}$ so that the degree of polynomials $n_{1}^{\prime \prime}$ and $n_{2}^{\prime \prime}$ is dropped down to $N p$-3, i.e., so that the coefficients $n_{1,(N p-1)}^{\prime \prime}, n_{1,(N p-2)}^{\prime \prime}, n_{2,(N p-1)}^{\prime \prime}$, and $n_{2,(N p-2)}^{\prime \prime}$ vanish

$$
\begin{aligned}
c_{1} & =n_{1,(N p-1)}^{\prime} / d_{(N p-2)}^{\prime} \\
c_{2} & =n_{2,(N p-1)}^{\prime} / d_{(N p-2)}^{\prime} \\
j b_{1} & =\left(n_{1,(N p-2)}^{\prime}-c_{1} d_{(N p-3)}^{\prime}\right) / d_{(N p-2)}^{\prime} \\
j b_{2} & =\left(n_{2,(N p-2)}^{\prime}-c_{2} d_{(N p-3)}^{\prime}\right) / d_{(N p-2)}^{\prime} .
\end{aligned}
$$

It can be shown that the polynomial $d^{\prime \prime}$ of the remainder network $N_{\mathbf{3}}$ as computed with (7) is of degree $\mathrm{Np}$-2. Since the degree of polynomials $n_{1}^{\prime \prime}, n_{2}^{\prime \prime}$, and $p^{\prime \prime}$ is $N p-3$, the network $\boldsymbol{N}_{\mathbf{3}}$ is actually a "reduced" version of the original network $\mathbf{N}$. The synthesis procedure can then continue by reiterating the two steps just described, obtaining the topologies in Fig. 1. Note that for $N p$ odd, the network $N_{3}$ at last step reduces to a simple network of degree one.

It is worth noting that in the most general case, in view of the fact that (6) is a system of nonlinear equations, the asymmetric lattice network is not unique for a given response. In fact, at each synthesis step, if $\left(J_{x 1}, J_{x 2}\right)$ is a solution of (6) then $\left(1 / J_{x 1}, 1 / J_{x 2}\right)$ is also a solution.

\section{B. Special Case: Derivation of Cul-De-Sac Topology}

The case where $n_{1}=n_{2}=n$ is of special interest, since it often occurs in the design of standalone filters. In fact, under this condition, (6) imposes $J_{x 1}=-J_{x 2}= \pm 1$, so (5) becomes

$$
\begin{aligned}
n_{1}^{\prime} & =2(n \mp p) \\
n_{2}^{\prime} & =2(n \pm p) \\
P^{\prime} & =0 \\
d^{\prime} & =4 d .
\end{aligned}
$$

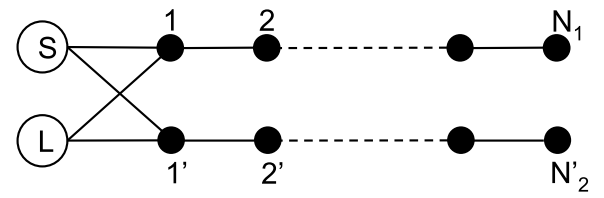

Fig. 3. Lattice network for $n_{1}(s)=n_{2}(s)$.

Since $P^{\prime}=0$, the network $N_{\mathbf{3}}$ is comprised of two disjoined one-port networks of admittances $Y_{1}(s)=n_{1}^{\prime} / d^{\prime}$ and $Y_{2}(s)=$ $n_{2}^{\prime} / d^{\prime}$ that are best synthesized separately. It can be shown that all the roots of $d^{\prime}$ are also the roots of the product $n_{1}^{\prime} \cdot n_{2}^{\prime}$. Cancellation will then occur in $Y_{1}(s)$ and $Y_{2}(s)$ in such a way that the sum of the degrees $N_{1}$ and $N_{2}$ of $Y_{1}(s)$ and $Y_{2}(s)$, respectively, is exactly $N p$. The resulting topology is one of the possible forms of Cul-de-sac topology and is shown in Fig. 3.

This form of the cul-de-sac can accommodate up to $N p$-1 transmission zeros ( $N p$ with the $S$ - $L$ coupling present).

From (3), it can be seen that the condition $n_{1}(s)=n_{2}(s)$ implies $(-1)^{N p} F^{*}=F$, and that is verified only if the roots of $F(s)$ are imaginary or in para-conjugate pairs. As a consequence, the asymmetric lattice always degenerates into the cul-de-sac for isolated filters exhibiting the generalized Chebyshev characteristic (the statement at the end of the Section II introduction is then demonstrated).

As a further consequence of this result, filters with complex reflection zeros arbitrarily placed (e.g., those constituting the channels in multiplexers) cannot be synthesized in cul-de-sac form. If the generation procedure in [1] (based on matrix rotations of the folded prototype coupling matrix) is applied to these filters, the asymmetric lattice topology is instead obtained.

\section{Synthesis of A CANONiCAL Prototype With FREQUENCY-DEPENDENT COUPLINGS}

The lattice network (Fig. 1) can be further manipulated to provide alternative realizations. Let $M$ and $C$ be the coupling and capacitance matrices, respectively, of the synthesized filter in Fig. 1(a). We assume that the admittance levels of the network have been normalized to unity, i.e., the diagonal of matrix $C$ contains only ones as nonzero elements (excluded first and last elements that are zero). Referring to Fig. 1(a) $(N p=10)$, a rotation with pivot $(1, N p)$ and angle $\alpha_{1}=-\tan ^{-1}\left(M_{S, N p} / M_{S, 1}\right)$ can be performed to annihilate coupling $(S, N p)$. Similarly, a rotation with the same pivot $(1, N p)$ and angle $\alpha_{2}=\tan ^{-1}\left(M_{1, L} / M_{N p, L}\right)$ can be performed to annihilate coupling $(L, 1)$. In both the cases, the coupling $(1, N p)$ will be generated. In general, the two angles $\alpha_{1}$ and $\alpha_{2}$ are different and both the two couplings cannot be eliminated simultaneously. This result can be, however, obtained with a further manipulation of $M$.

Let us assume in fact to multiply the capacitance of node 1 by the following value:

$$
C_{1}^{\prime}=-\frac{M(S, N p) M(N p, L)}{M(S, 1) M(1, L)} .
$$

In order to maintain the response unchanged, row and column 1 of $M$ must be multiplied by $\sqrt{C_{1}^{\prime}}$ obtaining the 


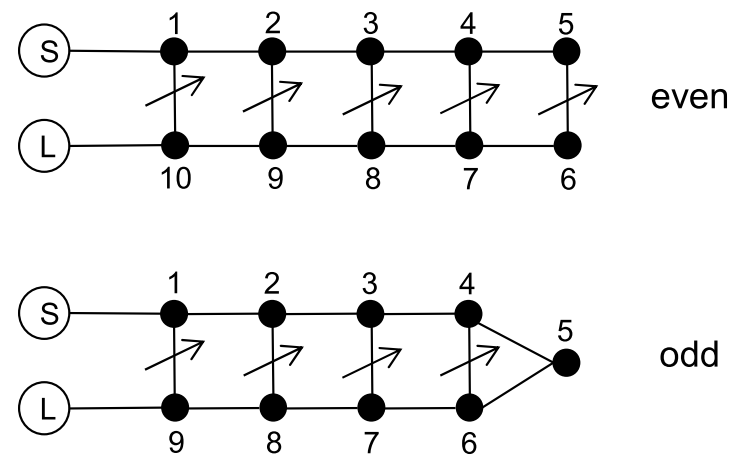

Fig. 4. Folded topology with frequency-variant couplings.

matrix $M^{\prime}$. Now, computing $\alpha_{1}$ and $\alpha_{2}$ from the element of $M^{\prime}$, we get $\alpha_{1}=\alpha_{2}=\alpha$ and rotation of $M^{\prime}$ with pivot $(1, N p)$ and angle $\alpha$ will annihilate both couplings $(S, N p)$ and $(L, 1)$ of matrix $M^{\prime}$ simultaneously. Of course, the same rotation needs to be carried over $C$ as well, producing the matrix $C^{\prime}$ which includes a frequency-dependent coupling (positive or negative) in position $(1, N p)$.

The above-mentioned operations can be reiterated $N p / 2$ times by assigning $M^{\prime} \rightarrow M$ and $C^{\prime} \rightarrow C$. The resulting networks for $N p$ even and odd are shown in Fig. 4, where the frequency-dependent couplings are marked with an arrow and are constituted by admittance inverters with parameter $J_{i, j}=M_{i, j}+\Omega \cdot C_{i, j}$.

It must be observed that not all the transversal couplings are necessarily frequency-variant. Some of them might be constant or might be even absent (depending on the imposed number of transmission zeros). Moreover, the sign of a frequency variant couplings $C_{i j}$ can be changed by multiplying row and column $i$ or $j$ of $M$ and $C$ by -1 .

\section{EXAMPLES}

The first example here reported concerns the nonfeasibility of cul-de-sac form in the case of the filters in a multiplexer. Let us consider the following diplexer specification in the lowpass normalized domain ( $R L$ is the passband return loss):

$$
\begin{aligned}
R x: B & =[-1,-0.084] \quad R L=20 \mathrm{~dB} N p_{r x}=6 \\
f z n_{r x} & =[.4337 i, .6906 i] \\
T x: B & =[0.4094,1] \quad R L=20 \mathrm{~dB} N p_{t x}=5 \\
f z n_{t x} & =[-.4122 i,-.111 i] .
\end{aligned}
$$

The characteristic polynomials of star-junction diplexer have been evaluated using the procedure illustrated in [19], assuming the shunt connection of the two filters at the input port. The filters are then synthesized with the folded topology and transformed with the rotations sequence described in Section II. The form finally obtained (Fig. 5) is the asymmetric lattice being the cul-de-sac not permitted in this case (reflection zeros not imaginary). The response of the diplexer is shown in Fig. 6.

The diplexer can be transformed in order to include frequency-variable couplings. To this end, we have applied the transformation explained in Section III to $M$ and $C$ matrices of both filters. The topology becomes that reported in Fig. 7 (the response remains the same as shown in Fig. 6).

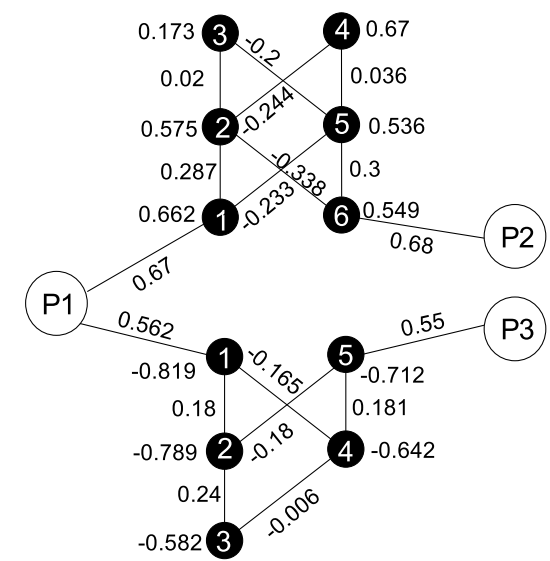

Fig. 5. Diplexer with asymmetric lattice filters. Numbers represent the elements of the coupling matrices of the two filters obtained after the rotation from (2).

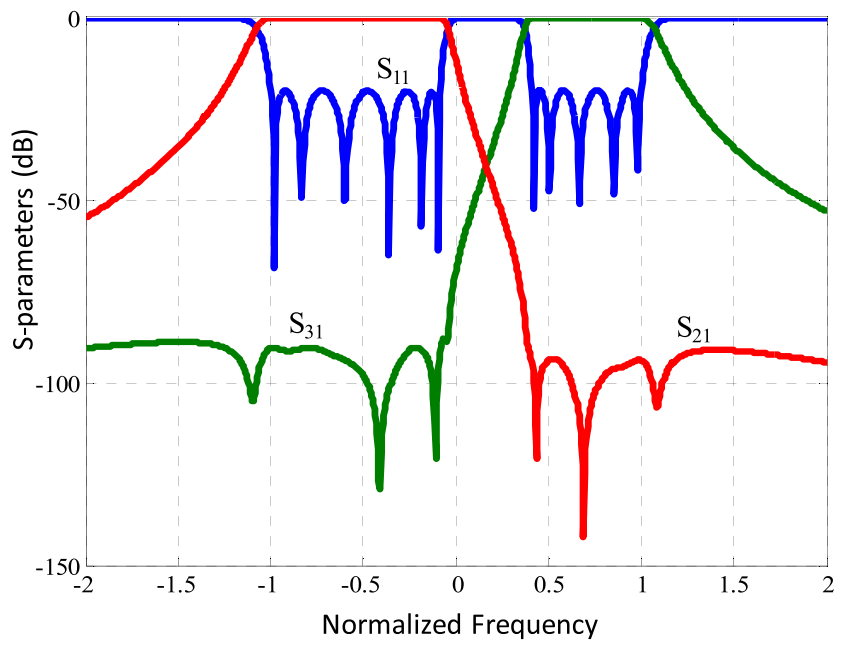

Fig. 6. Response of the synthesized diplexer.

The second example is taken from [8, Example 3]. It refers to an isolated filters with $N p=5, R L=20 \mathrm{~dB}$, and $f z=[-2.3 i,-1.5 i, 1.2 i, 1.8 i]$. In this case, the synthesis of the asymmetric lattice produces the cul-de-sac form [Fig. 8(a)], which is transformed into the folded form with two frequencydependent couplings [Fig. 8(b)] when the transformation of Section III is applied.

Note that, the elements of matrices $C$ and $M$ in Fig. 8(b) are practically coincident with those reported in [8, Table I]. The frequency response is not reported here, because it is coincident with that in [8, Fig. 3].

The last example shows how the synthesis of a prototype with frequency-dependent couplings can be carried out with the proposed approach also with complex zeros (for equalizing purpose). The filter data are taken from [12]: $N p=5$, $R L=20 \mathrm{~dB}$, and $f z=[ \pm 2 i, \pm 1.5]$. The final synthesized prototype is shown in Fig. 9, together with the frequency response (transmission and group delay). It can be observed that all the nodes have zero susceptance, due to the symmetry of the imposed transmission zeros. Moreover, the frequencydependent couplings in this case vanish at the center of the frequency characteristic (i.e., they resonate at the passband center frequency in the denormalized frequency domain). 


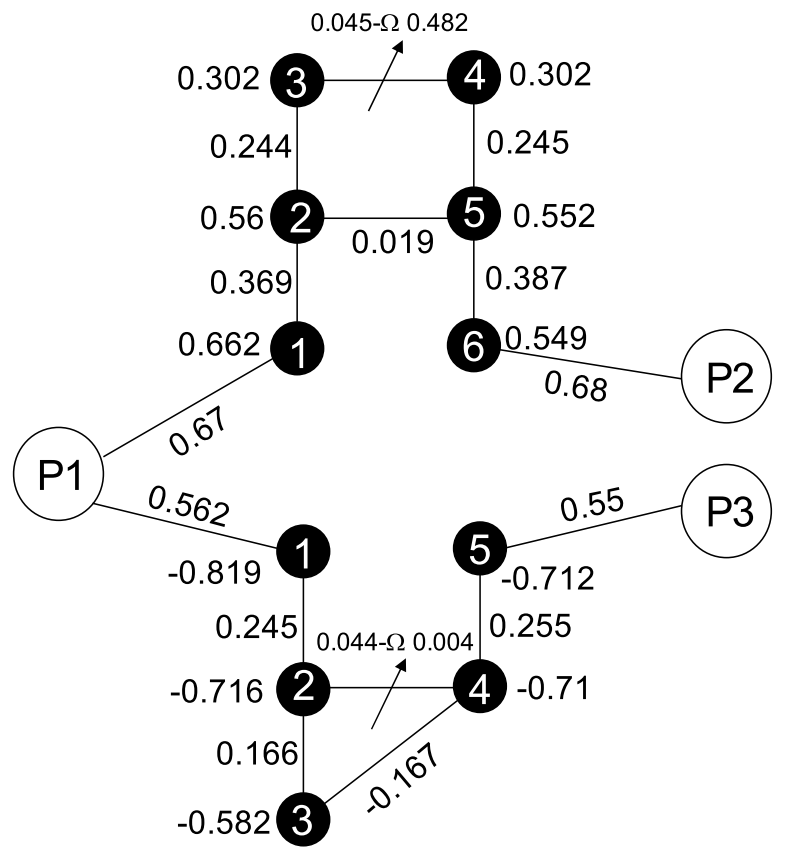

Fig. 7. Diplexer synthesized with frequency-variant couplings.

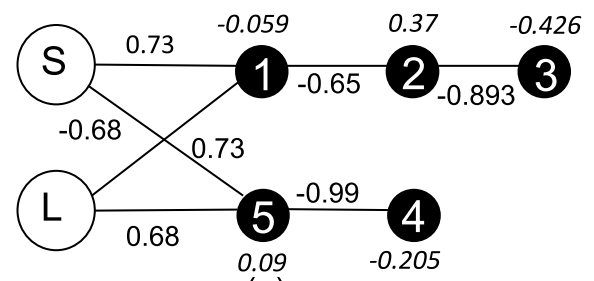

(a)

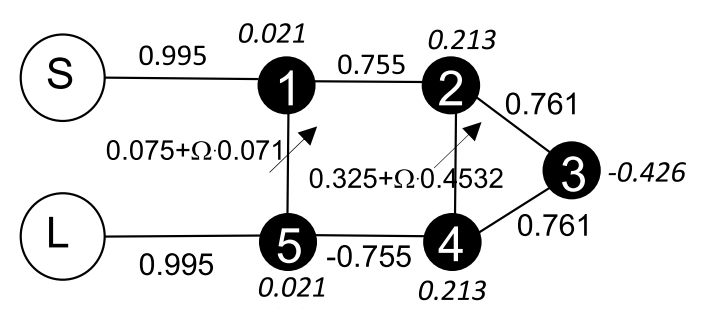

(b)

Fig. 8. Synthesis of filter from [8, Example 3]. (a) Cul-de-sac obtained from the asymmetric lattice. (b) Transformed topology with frequency-dependent couplings.

We can also observe that the network in Fig. 9(a) does not coincide topologically with that reported in [12, Fig. 1], which includes three frequency-dependent couplings; the two networks are, however, equivalent, because they exhibit the same response (generalized Chebyshev characteristic) and a suitable transformation should exist for transforming one into the other (this subject, however, needs further investigations).

\section{NOTES ON IMPLEMENTATION OF \\ FREQUENCY-DEPENDENT COUPLINGS}

After the synthesis of the prototype, we need to denormalize the circuit in order to derive suitable parameters for dimensioning the physical structure implementing the filter. We assume that the band-pass domain $f$ is related to the normalized low-pass domain $\Omega$ by the well-known expression $f_{n}=\left(f / f_{0}-f_{0} / f\right) / B_{n}$, with $f_{0}$ and $B_{n}$ center frequency and normalized bandwidth of the filter, respectively. For all

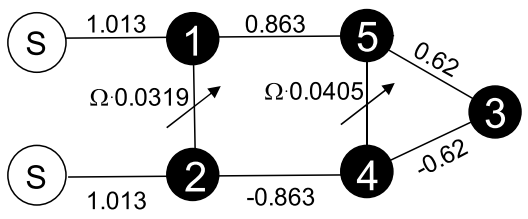

(a)

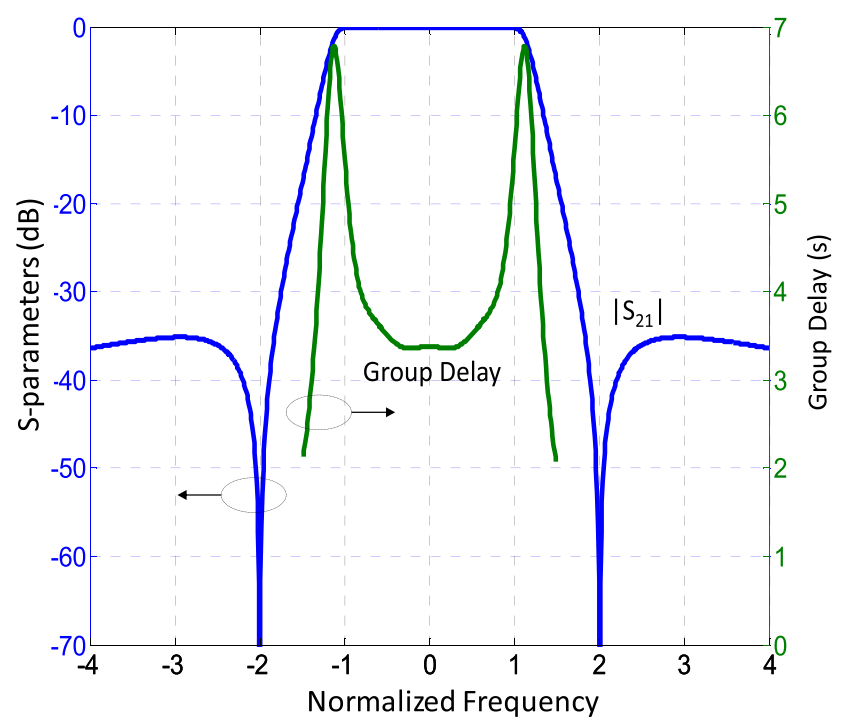

(b)

Fig. 9. Synthesized filter for the last example. (a) Routing scheme. (b) Transmission $\left(S_{21}\right)$ and group delay response.

the resonators and frequency-invariant couplings, the usual formulas expressing the coupling coefficients, the external $Q$ values, and the resonating frequencies as function of the elements of matrix $\mathbf{M}$ can be used [2]

$$
\begin{aligned}
f_{0, i} & =f_{0}\left[\sqrt{1+\left(\frac{M_{i, i} \cdot B_{n}}{2}\right)^{2}}-\left(\frac{M_{i, i} \cdot B_{n}}{2}\right)\right] \\
k_{i, j} & =M_{i, j} \cdot B_{n} \\
Q_{\mathrm{EXT}, S} & =\frac{1}{\left(M_{s, 1}\right)^{2} \cdot B_{n}} \\
Q_{\mathrm{EXT}, L} & =\frac{1}{\left(M_{N, L}\right)^{2} \cdot B_{n}} .
\end{aligned}
$$

For the frequency-dependent couplings, the denormalized admittance inverters are given by

$$
\bar{J}_{i, j}=M_{i, j}+\frac{C_{i, j}}{B_{n}}\left(\frac{f}{f_{0}}-\frac{f_{0}}{f}\right) .
$$

Unit capacitance resonators and unit loads have been assumed. Dividing $\bar{J}_{i, j}$ by the equivalent susceptance of the resonators $\left(1 / B_{n}\right)$, we get the frequency-variant coupling coefficient $\bar{k}_{i, j}$

$$
\begin{aligned}
\bar{k}_{i, j} & =B_{n} \cdot \bar{J}_{i, j} \\
& =B_{n} \cdot M_{i, j}+C_{i, j}\left(\frac{f}{f_{0}}-\frac{f_{0}}{f}\right) \\
& \cong k_{0, i . j}+2 C_{i, j}\left(\frac{f}{f_{0}}-1\right) .
\end{aligned}
$$




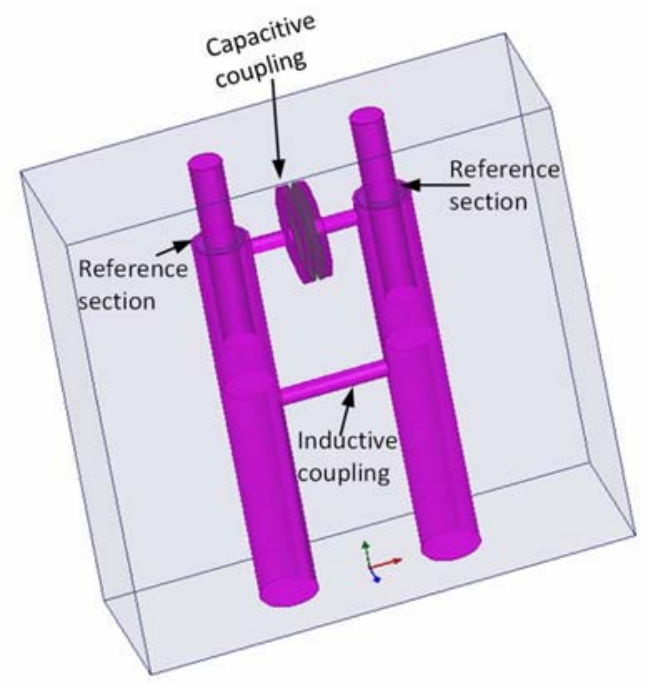

Fig. 10. Example of coupled-resonator structure implementing a frequencydependent coupling [21], [22].

In practice, we need to look for a physical coupling structure producing a coupling coefficient at frequency $f_{0}$ equal to $k_{0, i \cdot j}$ and vanishing at $f_{z}=f_{0}\left(1-k_{0, i, j} / 2 C_{i, j}\right)$. This result can be achieved by means of a suitable mix of electrical and magnetic couplings as shown in the literature in the case of coaxial coupled resonators [20], [21]. An example is reported in Fig. 10, where additional inductive and capacitive couplings are introduced in two coupled comb resonators.

The evaluation of the $Y$ parameters at the reference sections indicated in Fig. 10 allows the extraction of the frequencyvariant coupling parameters (using a suitable EM software like HFSS). In fact, from $y_{11}$ and $y_{12}$, we can compute $\bar{k}_{1.2}$ as follows:

$$
\bar{k}_{1,2}=\frac{2}{f_{0}} \frac{\operatorname{Im}\left(y_{12}\right)}{\partial\left(\operatorname{Im}\left(y_{11}\right)\right) / \partial f} .
$$

Fig. 11 shows $\bar{k}_{1.2}$ and $\operatorname{Im}\left(y_{11}\right)$ versus frequency for the structure in Fig. 10, from which we can derive $f_{0}=$ $724.07 \mathrm{MHz}, f_{z}=776.3 \mathrm{MHz}, k_{0,1,2}=0.0454$, and $C_{1,2}=$ $-k_{0,1,2} / 2\left(f z / f_{0}-1\right)=-0.0397$.

For dimensioning the filter structure, once the denormalized parameters have been computed, the dimensions affecting the couplings are adjusted until the extracted parameters coincide with those computed from the denormalized circuit.

Using the described approach, a diplexer for mobile communications has been designed and fabricated. It is used in dual band base station antennas to combine the two input signals (one at $700 \mathrm{MHz}$ and one at $800 \mathrm{MHz}$ in this case) onto the same radiating element. When independent electric tilt between the two bands is required, both input signals are first split in several copies, which are then individually phase-shifted and lastly combined in pairs onto the radiating elements. According to this, the number of diplexers in one such antenna can be as high as ten or more, and thus the need of compact filters (as those using inline structures) is evident.

The following specs have been assumed (return loss is $23 \mathrm{~dB}$ for both channels).

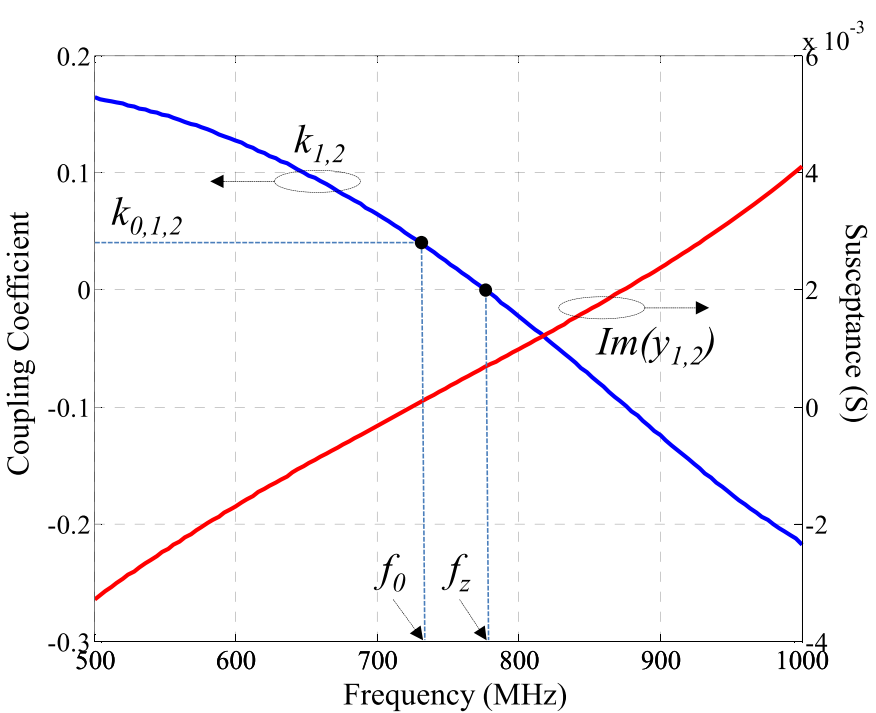

Fig. 11. Computed parameters for the structure in Fig. 10. The admittance parameters have been obtained by means of HFSS.

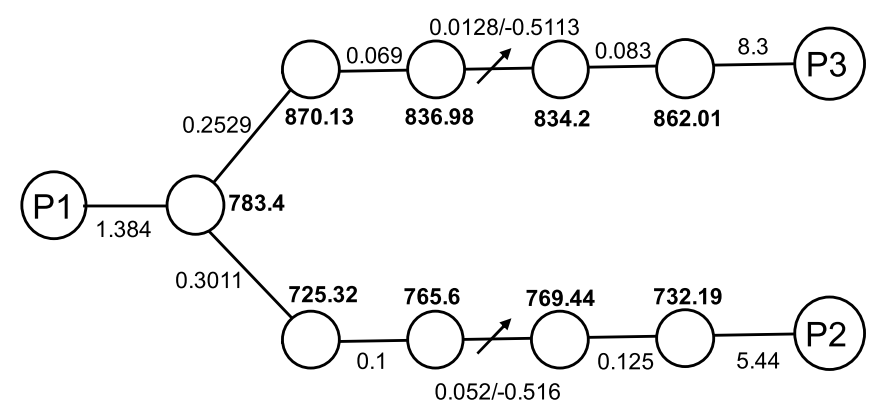

Fig. 12. Denormalized schematic of the diplexer. White circles represent the resonators (bold numbers are the resonating frequencies in megahertzes). Numbers next to the lines connecting the circles represent coupling coefficients or external $Q$ (this latter when the ports $P_{i}$ are involved). For frequencydependent couplings the pair $k_{0 i, j} / C_{i, j}$ is reported.

1) Junction: Resonant node.

2) $R X$ Filter: Band $=[683.3791 .2] \mathrm{MHz}$ and $f_{z}=$ 825.9 MHz.

3) $T X$ Filter: Band $=[818.2902 .1] \mathrm{MHz}$ and $f_{z}=$ $795 \mathrm{MHz}$.

The synthesis of the folded prototype of each filter is first carried out with the procedure outlined in Section III (characteristic polynomials of the diplexers are evaluated as described in [19]); then, applying (11)-(13), the denormalized circuit in Fig. 12 is obtained (resonating frequencies and coupling coefficients are reported. The frequency-dependent couplings are identified by the pair $k_{0 i, j} / C_{i, j}$ introduced previously). Note the inline topology of the filters, where the frequencydependent coupling allows the introduction of one transmission zeros (an inline filter with one or two transmission zeros obtained with constant couplings is shown in [22], but it is not practical for diplexers application).

The computed scattering parameters of the denormalized network are shown in Fig. 13 (lumped resonators and ideal inverters are assumed).

The diplexer has been then fabricated in comb configuration similar to that reported in Fig. 10. Fig. 14 shows a picture 


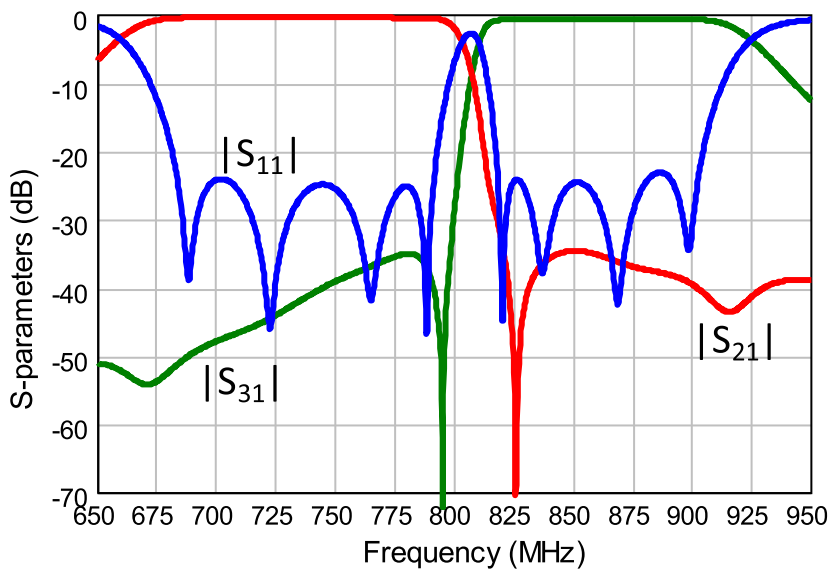

Fig. 13. Simulated response of the designed diplexer (denormalized network with ideal elements).

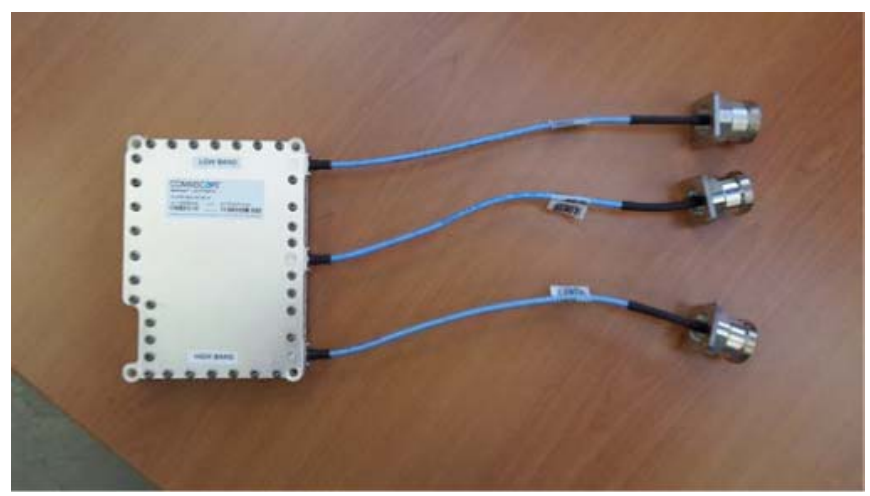

Fig. 14. Fabricated diplexer.

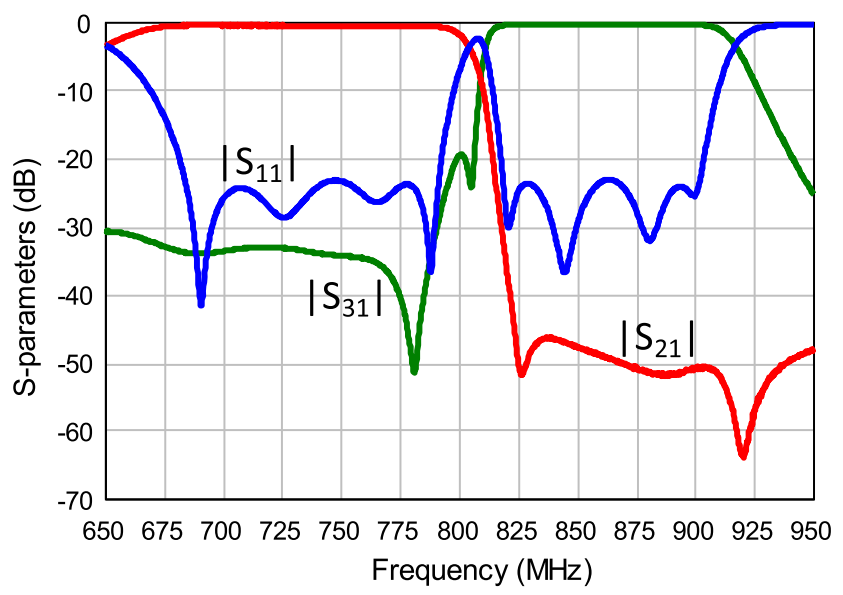

Fig. 15. Measured response of the fabricated diplexer.

of the realized device, whose dimensions are $154 \mathrm{~mm} \times$ $110 \mathrm{~mm} \times 24 \mathrm{~mm}$. The measured response is shown in Fig. 15, which is in reasonable agreement with the expected one (Fig. 13); the discrepancies (e.g., the additional zero in the measured $\left|S_{31}\right|$ ) are likely due to the actual frequency variation of all the couplings and to the spurious couplings in the implemented structure).

\section{CONCLUSION}

We have presented, in this paper, an original approach to the synthesis of microwave filters with frequency-dependent couplings. As pointed out at the beginning of this paper, the use of frequency-dependent couplings is convenient for reducing the overall size of filters maintaining the high selectivity allowed by the presence of transmission zeros. To this regard, this class of filters is of particular interest in applications, where the miniaturization is a primary requirement.

To the best of our knowledge, it is the first time that an explicit synthesis procedure, not exploiting optimization, has been proposed for the design of filters with frequencydependent couplings. We have also introduced a new type of canonical prototype with frequency-independent couplings (the asymmetric lattice), demonstrating that this topology degenerates into the cul-de-sac when the assigned reflection zeros are para-conjugate (imaginary or in pairs with opposite real part). The synthesized network with frequency-dependent couplings exhibits a folded topology with cross couplings linearly variable in the normalized frequency domain. We have then discussed the denormalization problem, explaining how to approach the dimensioning of the physical structures implementing the frequency-dependent couplings. Finally, we have verified the novel design approach by means of an experimental test device (diplexer for mobile communications).

Also the limitations of the considered class of filters should be mentioned in these conclusions. In our opinion, the main drawback is represented by the practical implementation of couplings presenting the required frequency dependence. In this paper, we have proposed a possible (approximate) solution in the case of coupled coaxial cavities. Other examples are reported in the literature [8]-[13] but further research work is needed to allow a wider practical use of these filters in the future.

\section{REFERENCES}

[1] R. J. Cameron, C. M. Kudsia, and R. Mansour, Microwave Filters for Communication Systems: Fundamentals, Design and Applications. Hoboken, NJ, USA: Wiley, 2007.

[2] D. Swanson and G. Macchiarella, "Microwave filter design by synthesis and optimization," IEEE Microw. Mag., vol. 8, no. 2, pp. 52-69, Apr. 2007.

[3] S. Amari and G. Macchiarella, "Synthesis of inline filters with arbitrarily placed attenuation poles by using nonresonating nodes," IEEE Trans. Microw. Theory Techn., vol. 53, no. 10, pp. 3075-3081, Oct. 2005.

[4] S. Amari, M. Bekheit, and F. Seyfert, "Notes on bandpass filters whose inter-resonator coupling coefficients are linear functions of frequency," in IEEE MTT-S Int. Microw. Symp. Dig., Atlanta, GA, USA, Jun. 2008, pp. $1207-1210$.

[5] W. Meng, H.-M. Lee, K. A. Zaki, and A. E. Atia, "Synthesis of multicoupled resonator filters with frequency-dependent couplings," in IEEE MTT-S Int. Microw. Symp. Dig., May 2010, pp. 1716-1719.

[6] S. Amari and J. Bornemann, "Using frequency-dependent coupling to generate finite attenuation poles in direct-coupled resonator bandpass filters," IEEE Microw. Guided Wave Lett., vol. 9, no. 10, pp. 404-406, Oct. 1999.

[7] M. Politi and A. Fossati, "Direct coupled waveguide filters with generalized Chebyshev response by resonating coupling structures," in Proc. Eur. Microw. Conf. (EuMC), Sep. 2010, pp. 966-969.

[8] L. Szydlowski, A. Lamecki, and M. Mrozowski, "Coupled-resonator filters with frequency-dependent couplings: Coupling matrix synthesis," IEEE Microw. Wireless Compon. Lett., vol. 22, no. 6, pp. 312-314, Jun. 2012.

[9] L. Szydlowski, N. Leszczynska, A. Lamecki, and M. Mrozowski, "A substrate integrated waveguide (SIW) bandpass filter in a box configuration with frequency-dependent coupling," IEEE Microw. Wireless Compon. Lett., vol. 22, no. 11, pp. 556-558, Nov. 2012. 
[10] L. Szydlowski, A. Lamecki, and M. Mrozowski, "Coupled-resonator waveguide filter in quadruplet topology with frequency-dependent coupling-A design based on coupling matrix," IEEE Microw. Wireless Compon. Lett., vol. 22, no. 11, pp. 553-555, Nov. 2012.

[11] L. Szydlowski, A. Jedrzejewski, and M. Mrozowski, "A trisection filter design with negative slope of frequency-dependent crosscoupling implemented in substrate integrated waveguide (SIW)," IEEE Microw. Wireless Compon. Lett., vol. 23, no. 9, pp. 456-458, Sep. 2013.

[12] L. Szydlowski and M. Mrozowski, "A self-equalized waveguide filter with frequency-dependent (resonant) couplings," IEEE Microw. Wireless Compon. Lett., vol. 24, no. 11, pp. 769-771, Nov. 2014.

[13] L. Szydlowski, N. Leszczynska, A. Lamecki, and M. Mrozowski, "Dimensional synthesis of coupled-resonator pseudoelliptic microwave bandpass filters with constant and dispersive couplings," IEEE Trans. Microw. Theory Techn., vol. 62, no. 8, pp. 1634-1646, Aug. 2014.

[14] J. T. Kuo, Y. C. Wang, and J. W. Kuo, "Diplexer with trisections synthesized by frequency-dependent coupling," in Proc. Asia-Pacific Microw. Conf. (APMC), vol. 3. Dec. 2015, pp. 1-3.

[15] S. Amari, "On the maximum number of finite transmission zeros of coupled resonator filters with a given topology," IEEE Microw. Guided Wave Lett., vol. 9, no. 9, pp. 354-356, Sep. 1999.

[16] S. Tamiazzo and G. Macchiarella, "A canonical prototype for coupledresonator filters with frequency-dependent couplings," in IEEE MTT-S Int. Microw. Symp. Dig., San Francisco, CA, USA, May 2016, pp. 1-3.

[17] H. C. Bell, Jr., "Canonical asymmetric coupled-resonator filters," IEEE Trans. Microw. Theory Techn., vol. MTT-30, no. 9, pp. 1335-1340, Sep. 1982.

[18] R. J. Cameron, A. R. Harish, and C. J. Radcliffe, "Synthesis of advanced microwave filters without diagonal cross-couplings," IEEE Trans. Microw. Theory Techn., vol. 50, no. 12, pp. 2862-2872, Dec. 2002.

[19] G. Macchiarella and S. Tamiazzo, "Novel approach to the synthesis of microwave diplexers," IEEE Trans. Microw. Theory Techn., vol. 54, no. 12, pp. 4281-4290, Dec. 2006.

[20] H. Wang and Q. X. Chu, "An inline coaxial quasi-elliptic filter with controllable mixed electric and magnetic coupling," IEEE Trans. Microw. Theory Techn., vol. 57, no. 3, pp. 667-673, Mar. 2009.

[21] S. Bastioli, R. Snyder, and P. Jojic, "High power in-line pseudoelliptic evanescent mode filter using series lumped capacitors," in Proc. 41st Eur. Microw. Conf. (EuMC), Oct. 2011, pp. 87-90.

[22] G. Macchiarella, "Synthesis of an in-line prototype filter with two transmission zeros without cross couplings," IEEE Microw. Wireless Compon. Lett., vol. 14, no. 1, pp. 19-21, Jan. 2004. 